\title{
Coherence of retrieval cues, rather than additivity, determines prior cuing effectiveness in the rat
}

\author{
PASCALE GISQUET-VERRIER \\ Laboratoire de Neurobiologie de l'Apprentissage et de la Mémoire, C.N.R.S., URA 1491, Université \\ Paris Sud, Orsay, France
}

\begin{abstract}
In previous studies, we have shown that adding a supplementary cue in a prior-cuing treatment may dramatically reduce its initial effectiveness. This series of experiments was aimed at testing the hypothesis that such a lack of additivity among retrieval cues could be due to the combined reactivation of memory attributes related to incompatible behavioral responses. For that purpose, rats were partially trained to reach the lighted arm (light condition; usual training condition) or the darkened arm (dark condition) of a Y-maze in order to avoid an electric footshock. The first experiment demonstrates that animals learned the two versions of the task similarly and that an exposure to the light similarly acts as an effective retrieval cue after a 24-h interval. In the second experiment, the effectiveness of exposure to the grid alone and to the light alone as well as to the combined light + grid cue was investigated after a 21-day training-to-test interval in the light condition, where light was related to safety and grid was associated with the shock, and in the dark condition, where both light and grid were related to the shock. The results indicate that whereas the compound light + grid cue was an ineffective retrieval cue in the light condition, it acted as an effective retrieval cue in the dark condition, supporting the hypothesis that coherence between retrieval cues is a necessary condition for effective cuing.
\end{abstract}

There is considerable evidence indicating that an exposure to some components of a learning episode can alleviate a performance decrement resulting from experimentally induced amnesia (Koppenaal, Jagoda, \& Cruce, 1967; R. R. Miller \& Springer, 1972; Sara, 1973a), spontaneous decrease in performance (Kamin effect: Klein \& Spear, 1970; Gisquet-Verrier \& Alexinsky, 1990a), short retention intervals (Gordon \& Feldman, 1978) or long-term forgetting (Deweer, Sara, \& Hars, 1980; Gisquet-Verrier \& Alexinsky, 1986; GisquetVerrier, Dekeyne, \& Alexinsky, 1989). The facilitative effect of prior cuing is a highly reliable phenomenon that has been found in many different species, such as rats, mice, and pigeons (Moye \& Thomas, 1982), as well as in human infants (Rovee-Collier \& Hayne, 1987). There are good arguments for asserting that the alleviation of forgetting by prior cuing is quite independent of any new learning. This is illustrated, for example, by experiments showing that prior cuing can be effective even when it entails extinction, which, if it occurred in other circumstances, would weaken the expression of that memory (Gordon, 1981; Sara, 1973b).

A memory trace can be considered as a collection of memory attributes, which are the internal representations of the different events noticed by the subject at the time of the initial episode, with each of them developing over time more or less independently (Spear, 1978; Under-

Correspondence should be addressed to P. Gisquet-Verrier, NAM, Université Paris Sud, Bat 446, 91405 Orsay Cedex, France. wood, 1969). The general view holds that the presentation of the cue activates the corresponding memory attribute(s). This reactivation may be able to trigger the reactivation of the other attributes linked with it or them, provided that the subject notices a sufficient number of events or an appropriate kind of event (Spear, 1978). This may then ultimately lead the whole memory trace to pass from a passive to an active state (Lewis, 1979).

A wide range of prior cuing treatments has been shown to be effective in alleviating forgetting. The most commonly used is noncontingent exposure to the unconditioned stimulus (typically a footshock; see Lewis, Misanin, \& R. R. Miller, 1968), but exposure to the conditioned stimulus (CS; see Gisquet-Verrier \& Alexinsky, 1990a), to the experimental context (Deweer, 1986), or even to the internal context (Richardson, Riccio, \& Axiotis, 1986) have also been shown to be effective. Since different retrieval cues can be expected to reactivate different numbers and types of attributes constituting the target memory, one may postulate that exposure to several training features may lead to the activation of a larger number of memory attributes and thus have a greater effect on retention performance than would exposure to a single feature. Direct comparison of the effects of several prior cuings in the same experiment are rare in the literature. The few existing examples show inconsistent results (Devietti \& Hopfer, 1974; Deweer, 1986; Moye \& Thomas, 1982).

Recently, we investigated the effects of nine pretest cuing treatments, after three particular retention intervals (Gisquet-Verrier et al., 1989). In these experiments, rats 
were trained to reach the lighted arm of a Y-maze for 15 massed trials in order to escape or avoid an electrical footshock delivered through the grid floor. At the end of training, the brightness discrimination was well-acquired, whereas the avoidance response was not. Retention performance, defined as the difference in avoidance between the beginning of testing and the end of training, was disrupted after a 1-h training-to-test interval (TTI), enhanced following a 3-day TTI and attenuated by spontaneous forgetting after 21 days (Gisquet-Verrier \& Alexinsky, 1988; Gisquet-Verrier \& Alexinsky,1990b). The effects of the nine cuing treatments on the retention performance were studied after these three retention intervals. Pretest cuing consisted of a 2.5 -min exposure to various isolated training features, such as the light that constituted the CS, the grid through which the electrical shock was delivered during initial training, the experimental context, and a combination of several training features: the grid plus the unconditioned stimulus (UCS), the CS plus the UCS, or a well-ordered sequence of events between CS and UCS. The cuing treatments ended $5 \mathrm{~min}$ before the retention test.

The results showed that after a given retention interval the retention performance can be improved following various pretest cuing treatments. The fact that reactivation of the memory trace can be promoted from the reactivation of different memory attributes provides experimental support for a multidimensional conception of the memory trace. It further indicates that the effectiveness of a particular cuing treatment may increase or decrease over time, suggesting that the memory trace is modified as a function of the length of the retention interval (see also J. S. Miller, Jagielo, \& Spear, 1991). After each of the studied retention intervals, it appears that the kind of presented features determines the cuing effectiveness more than does the number of presented features. Contrary to what was expected, no simple additivity rule between retrieval cues (i.e., that the greater the number of training cues included in the cuing treatment, the greater would be the effectiveness of the cuing treatment) operated. On the contrary, in some cases, the addition of a supplementary training feature dramatically reduced the effectiveness of a cuing treatment. More precisely, it was found that exposing the subjects to short flashes of light, mimicking the CS, considerably improved the retention performance $1 \mathrm{~h}$ following initial training. However, when the same treatment was applied to animals placed in the cuing box equipped with a grid similar to the one through which the electrical shock was delivered during initial training, the effectiveness of prior cuing was significantly reduced. After a 3-day TTI, at the time of a reminiscence-like phenomenon, no cuing treatment was able to further enhance the retention performance. Exposing the animals to a box equipped with the grid did not affect the retention performance. When additional exposures to the CS were delivered to the subjects in the cuing box equipped with the grid, the retention performance was significantly disrupted, relative to that of noncued animals. On the other hand, after a 21-day TTI, exposing the rats to the cuing box equipped with the grid significantly improved the retention performance. However, animals exposed to the CS plus the grid exhibited retention performance significantly worse than that of animals simply cued with the grid. Hence, it appears that the two features light and grid interact at the time of cuing. Under circumstances in which one of them is effective, adding the other dramatically reduced its effectiveness. Since, in our training situation, the grid was related to the footshock whereas the light indicated the safe place, we proposed that such results could be due to combined reactivation of incompatible parts of the initial memory (Gisquet-Verrier et al, , 1989).

An alternative explanation of the reduced effect of compound cue versus simple cue is that the added information distracts the animal, preventing it from paying sufficient attention to the information able to promote the retrieval processes. Such an interpretation was adopted by Deweer (1986) in one of the rare studies comparing the effectiveness of several retrieval cues. This author showed that exposure to the UCS (an appetitive reinforcer) and to the experimental context were able to alleviate the performance deficit due to long-term forgetting when delivered successively but not when delivered concurrently.

To investigate these two possibilities, the effectiveness of the light cue, the grid cue, and the compound light + grid cue was tested under two training situations: (1) our usual training condition in which animals were trained to reach the lighted arm of the maze, and where light and grid cues may refer to incompatible behavioral responses, and (2) another condition in which animals were trained to reach the darkened arm of the lighted maze, and where light and grid cues were both related to the shock, thus possibly referring to compatible behavioral responses. According to our interpretation, the compound cue will be less effective than the single cue only in the light condition and not in the dark condition. According to an interpretation in terms of disruption of attentional processes, the reduced effectiveness of the compound cue will be obtained in the light training condition and in the dark training condition.

Before directly addressing this question, it was important to demonstrate that the dark training condition was equivalent to the light one. Experiment 1 was aimed at testing whether or not animals learned the two versions of the task similarly and, more importantly for our purpose, also at determining whether or not an exposure to the light may act as an effective retrieval cue with a 1-h TTI in the dark condition, as has already been shown in the light condition (Gisquet-Verrier \& Alexinsky, 1990a).

Experiment 2 was more specifically aimed at testing our hypothesis concerning a possible detrimental effect of combining several retrieval cues. For this experiment, a 21-day retention interval was chosen in light of our previous results. This retention interval allows further comparison between the two versions of the brightness discrimination avoidance task. 


\section{GENERAL METHOD}

\section{Subjects}

The subjects were naive male Sprague-Dawley rats obtained from the Iffa-Credo rearing center. They were 50-57 days old and weighed $250 \mathrm{~g}$ at their arrival in the laboratory. They were housed in pairs in wire-mesh cages and had free access to food and water throughout the experiment.

\section{Apparatus}

Training and testing were carried out in a fully automated black Perspex Y-maze with arms $13 \mathrm{~cm}$ wide $\times 60 \mathrm{~cm}$ long $\times 38 \mathrm{~cm}$ high and a $25-\mathrm{cm}$ equilateral triangular choice area. A 40-W lamp was located at the end of each arm. The floor consisted of 3-mmdiam grids, spaced $9 \mathrm{~mm}$ apart. A shock generator (Campden, Model 521S), set to deliver scrambled shock, provided the incentive for learning. Photocells placed $1 \mathrm{~cm}$ (proximal), $11 \mathrm{~cm}$ (medial), and $30 \mathrm{~cm}$ (distal) from the entrance of each arm were connected to an Apple II microprocessor, which controlled the light sequence as well as the length of the intertrial interval and recorded latencies and the sequence of visited arms. The maze was housed in a darkened room, adjacent to the colony room.

\section{"L" CONDITION}
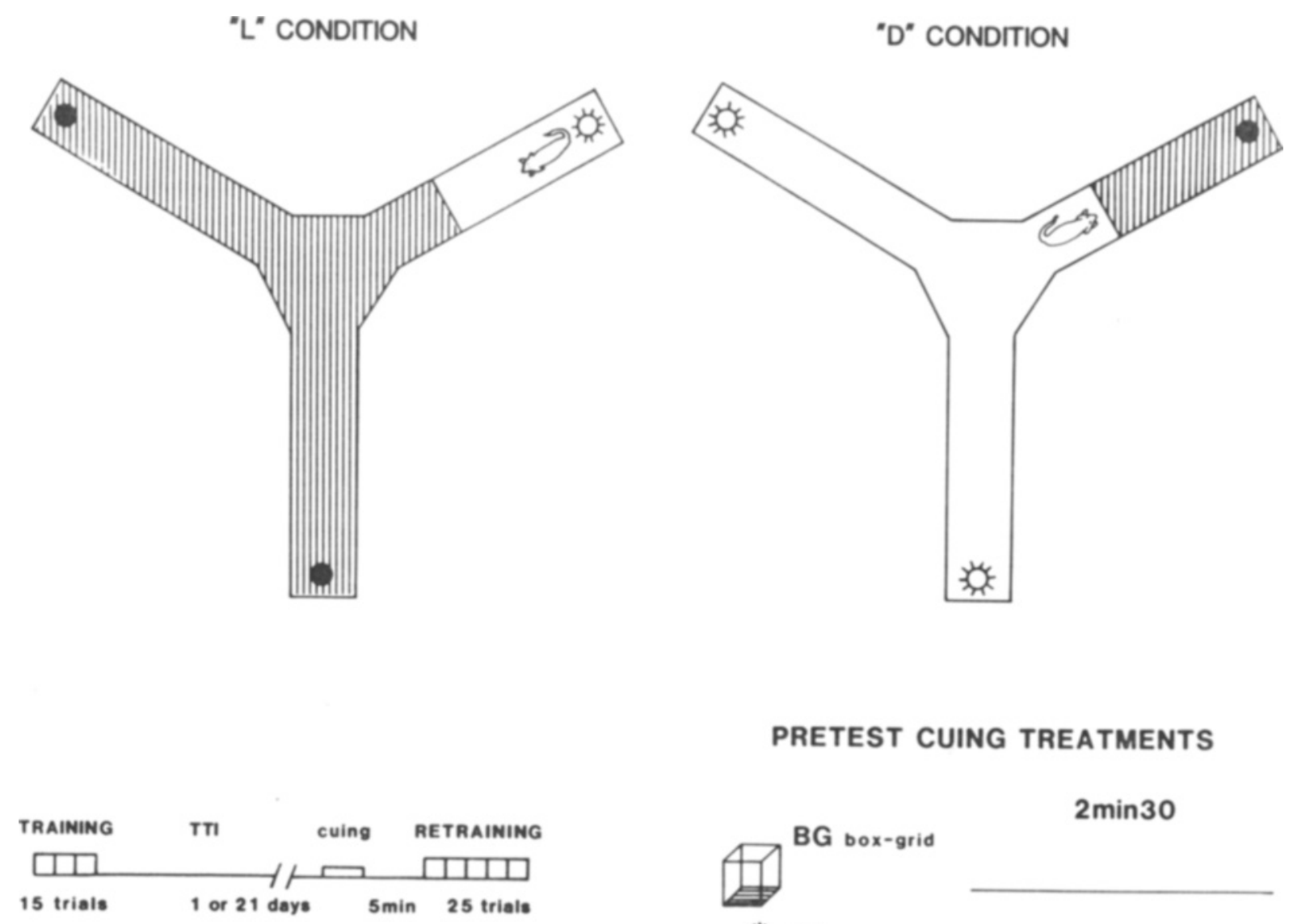

Cuing treatments occurred in a cuing box, which differed from the experimental apparatus in size $(23 \times 23 \times 36 \mathrm{~cm})$ and color (beige). The cuing box was covered by a removable ceiling equipped or not with a 40-W lamp. A removable grid floor identical to the floor of the Y-maze could be added in the cuing box.

\section{Procedure}

The general design of this series of experiments is illustrated in Figure 1. Two general training and testing situations were adopted. In each experiment, half of the animals were trained to reach the lighted arm of the darkened maze ( $L$ condition), whereas the other half were trained to reach the darkened arm of the lighted maze (D condition).

Pretraining. On Day 1, the rats, in groups of 6, were given 15-min free exploration in the entirely lighted maze ( $\mathrm{L}$ condition) or entirely darkened maze ( $\mathrm{D}$ condition). On Day 2 , each rat was placed in the maze (lighted or darkened) and allowed to explore the apparatus for $5 \mathrm{~min}$.

Training. Training began on the following day. All animals were given 15 training trials according to the following procedure: Each rat was placed in the start arm of the Y-maze for $20 \mathrm{sec}$, with the arm lighted in the $\mathrm{L}$ condition or darkened in the $\mathrm{D}$ condition. The
PRETEST CUING TREATMENTS

\section{$2 \min 30$}

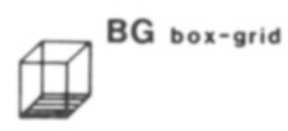

BL box-light

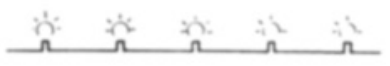

BGL box-grid-light

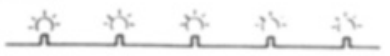

Figure 1. Experimental design. See text for explanations. 
door closing the start arm (and used only on the first trial) was then opened, the light was turned off, and another arm was lighted ( $\mathrm{L}$ condition), or the light was turned on and another arm was darkened (D condition). Five seconds later, an electric shock was applied to the grid floor in the whole maze, except for the terminal $30 \mathrm{~cm}$ of the safe arm. During the first five trials, the intensity of the electrical shock was adapted for each animal just below the squeak threshold and corresponded to the minimal level for eliciting a rapid response (between 0.3 and $0.5 \mathrm{~mA}$ ). The rat had to run to the safe arm in order to escape or to avoid the footshock.

The time elapsed from the lighting or the darkening of a new goal arm until the rat crossed the proximal photobeam of the initial arm was defined as the start latency. The time elapsed until the subject crossed the distal photobeam of the new goal arm was defined as the response latency. When the animal crossed the medial photobeam of an arm, this arm was considered as having been visited. An incorrect response was scored when the rat entered into one of the shocked alleys, before escaping into the safe alley. Animals that failed to respond within $60 \mathrm{sec}$ were manually pushed into the safe arm; a response latency of $60 \mathrm{sec}$ was then scored. The rat remained in the safe compartment for a 20 -sec intertrial interval, during which an exit from the safe alley led to shock. The goal alley on any given trial served as the start alley for the next trial. The sequence of correct right (R) and left (L) turns in the Y-maze was determined according to the following schedule: $L-L-R-L-R-$ R-L-R-R-L-R-L-L-R-R.

After completion of the 15 training trials, the rats were removed from the maze and returned to their home cages, where they remained for the TTI ( 1 day in Experiment 1 and 21 days in Experiment 2). Animals responding with mean latencies greater than $30 \mathrm{sec}$ during the last five training trials were discarded. In the light and dark training conditions, animals were divided into matched groups on the basis of their performance: response latencies, number of errors, and number of avoidance responses obtained during the whole training session (i.e., the three blocks of five trials).

Cuing. Before the testing session, animals in the cued groups were placed in the cuing box set in a room situated between the colony room and the experimental room for $2.5 \mathrm{~min}$. Three cuing conditions were studied in this series of experiments: During Treatment $B L$ (box + light), the subjects were exposed to a series of five 2 -sec flashes presented every $28 \mathrm{sec}$, similar (except for duration) to those used previously as the conditioned stimulus. Animals submitted to Treatment $B G$ (box + grid) were placed in the cuing box equipped with the grid. Treatment $B G L$ (box + grid + light) was a compound treatment: the animals were placed in the cuing box equipped with the grid and were additionally submitted to five $2-\mathrm{sec}$ illuminations occurring under the same circumstances as in treatment BL.

Motor activity of the animals during cuing was registered: During each of the five $30-\mathrm{sec}$ periods of the cuing treatment, horizontal activity was scored $0,1,2$, or 3 according to a global evaluation of the locomotor activity level. Vertical activity (i.e., the number of rearings) was also noted. The scores during the five $30-\mathrm{sec}$ periods of cuing were added; they constituted the horizontal and vertical activity scores for each animal. Motor activity was always registered by the same experimenter, who did not know the training condition to which the rat was assigned (see Gisquet-Verrier \& Alexinsky, 1990a). At the end of the cuing treatment, the animals returned to their home cage for a 5-min period.

Testing. At the end of this period for the cued condition or at the end of the retention interval for the noncued condition, the animals were removed from their home cages and carried to the experimental room. They were placed directly into the start arm. Twenty seconds later, the starting door was opened and the animals were retrained for 25 trials in the same conditions as during the initial training, with the lighted or darkened arm positioned ac- cording to the following sequence: $L-R-R-R-L-L-R-L-L-L-R-$ L-L-R-L-R-R-R-L-L-R-R-L-R-R.

Behavioral measures. Throughout training and testing, start latencies, response latencies, and sequences of visited arms were recorded for each trial. As already pointed out (Gisquet-Verrier \& Alexinsky, 1988), a real avoidance response is difficult to obtain in our training condition, considering the length of the CS-UCS interval $(5 \mathrm{sec})$ relative to the size of the apparatus, as well as the small number of training trials. A start latency below $5 \mathrm{sec}$ corresponded to an exit from the initial arm during the CS-UCS interval. Such a response, emitted before the onset of the UCS, roughly equivalent to an avoidance response in a shuttlebox apparatus, was considered as an attempt to avoid and was scored 1 . A response latency less than $5 \mathrm{sec}$ permitted avoidance of the shock and was scored 2. The response latencies (after a logarithmic transformation), the number of errors, and the avoidance score were pooled in blocks of five trials for training and testing. Number of errors was very low at the end of training so that this measure was not useful during retention because of a floor effect. Avoidance scores were preferred to response latencies as an index of learning the task. because starting to move before the onset of the UCS seemed to be more appropriate as an adaptive response than moving faster. Furthermore, a few escape responses with long latencies due to emotional reactiveness to shock (freezing) had a greater effect on the mean latencies than did several avoidance responses emitted within a response time close to $5 \mathrm{sec}$. Finally, it is considered that the performance obtained at the beginning of the retraining session better reflects the level of retention than does the entire reacquisition performance for which retrieval and reacquisition processes might interact. Therefore, savings in avoidance, expressed as the difference between the mean avoidance score of the first five testing trials and that of the last five training trials, served as a measure of retention (for more details, see Gisquet-Verrier \& Alexinsky, 1988).

Statistical analyses. Analyses of variance (ANOVAs) were performed on the data. Within-group comparisons compared performance obtained during the first five testing trials with that obtained during the last five training trials. Paired comparisons analyzed between-group differences concerning either the savings in avoidance or the number of avoidance responses (avoidance score) during testing.

The experiments were run over several consecutive days. Every day, animals of each of the experimental conditions were placed in the pretraining phase and then over subsequent days were subjected to the whole procedure until the different experimental groups ( $n=15$, in each group) were completed.

\section{EXPERIMENT 1}

Before determining whether light + grid would be less effective than grid in the dark and light training conditions, we had to verify that animals are able to perform similarly in the dark and light training versions of the task, as previously suggested by Ray and Barrett (1975). We had also to verify the effectiveness as a retrieval cue of an exposure to the light in the dark training condition. We repeatedly observed that, in the light training condition, a pretest exposure to the light delivered $1 \mathrm{~h}$ or 1 day after initial training induced a large enhancement of the subsequent retention performance. However, in the dark condition, the status of the light was partially modified, since lighted places were now related to the UCS. The light and dark training conditions could only be used to test our coherence hypothesis insofar as light similarly 
acted as a retrieval cue in both conditions (i.e., through its $\mathrm{CS}$ value).

\section{Method}

Sixty-one male Sprague-Dawley rats served as subjects in Experiment 1 . Thirty-one animals were trained in the light condition; the remaining 30 were trained in the dark condition. At the end of training, 1 animal was discarded because of its elevated run time during initial training. In each training condition, the animals were placed in two groups, on the basis of their training performance, according to a $2 \times 2$ factorial design ( $n=15$, in each group): noncued animals (Groups L-NC and D-NC) and cued animals (Groups L-BL and D-BL), which were exposed to the CS in the cuing box. After a 1-day TTI, the animals were either cued or directly placed into the Y-maze. All animals were retrained for 25 trials under the same conditions as those in initial training.

\section{Results}

Pretraining. During the 5-min pretraining period, animals placed in the darkened maze displayed a significantly higher score of visited arms than did animals placed in the lighted maze $[M=22.43$ vs. $M=19.60 ; F(1,56)=$ $6.05, p<.025]$.

Initial training. ANOVAs (training condition $\times$ trial block) indicated a significant effect of trial blocks $(p<.001$, for each considered variable) and no effect of the training condition $(F \mathrm{~s}<1)$. There was, however, a significant interaction between training conditions and trial blocks for response latencies and for errors $[F(2,116)=6.45, p<.005$, and $F(2,116)=4.70$, $p<.025$, respectively], but not for the number of avoidance responses (see Figure 2). Nevertheless, no differ-
"L" CONDITION

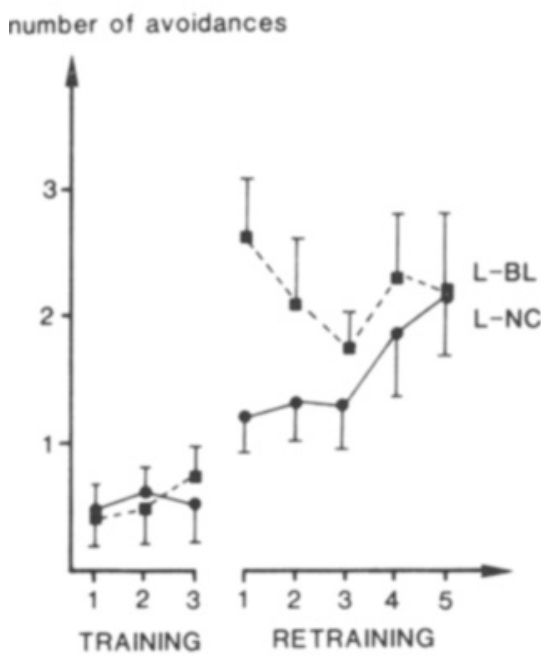

1 day TTI

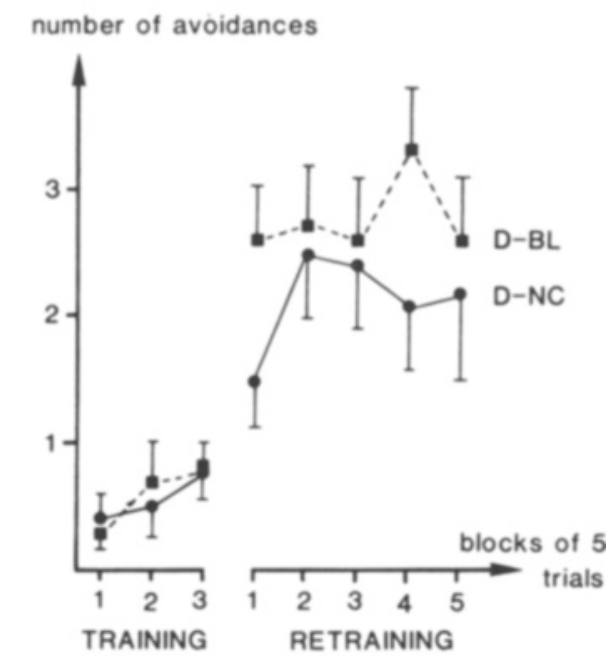

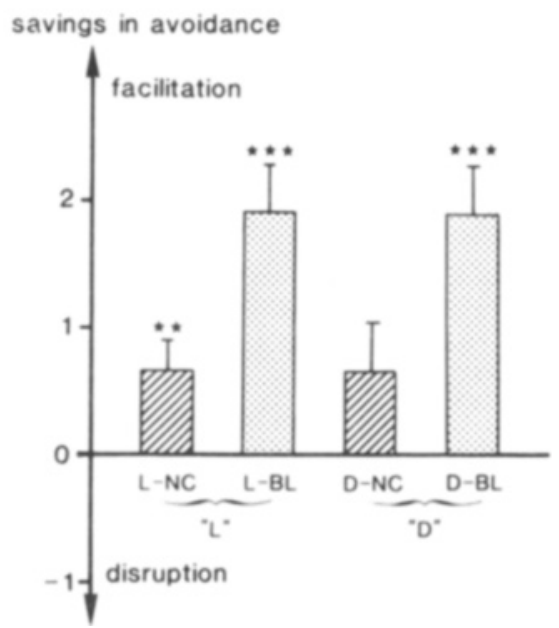

Figure 2. Upper: Mean number $( \pm S E M)$ of avoidance responses (avoidance score) obtained during training and retraining in the light $(\mathrm{L})$ and dark (D) training conditions for noncued animals (L-NC and D-NC) and animals cued with the light (L-BL and D-BL). Lower: Mean ( $\pm S E M)$ score of savings in avoidance (difference between the score obtained during the first five testing trials and the last five training trials) obtained for each group. Asterisks at the top of the columns correspond to within-group comparisons $\left(^{* *} .025<p<.10\right.$; ***p $<.001$ ). 
ence could be found at the end of training between $D$ and $\mathrm{L}$ animals, regardless of the considered variable $(F<1$, in every case).

Cuing. No difference in terms of activity during the 2.5 min of the cuing treatment could be detected between the two cued groups L-BL and D-BL.

Retraining. Mean number of avoidance responses during training and testing, as well as mean savings in avoidance, obtained for animals trained under $\mathrm{L}$ and $\mathrm{D}$ conditions are illustrated in Figure 2.

Effect of the training conditions $(\mathrm{D} / \mathrm{L})$. ANOVAs (training condition $\times$ trial blocks) revealed no significant difference between the $\mathrm{L}$ or $\mathrm{D}$ training conditions during the five blocks of testing trials, regardless of the considered variable: number of avoidance responses, response latencies, or number of errors.

Effect of pretest cuing. Within-group comparisons on the number of avoidance responses (avoidance score) indicated a significant improvement of performance between the end of training and the beginning of retraining for both cued groups L-BL $[F(1,14)=26.38, p<.001]$ and D-BL $[F(1,14)=23.95, p<.001]$, as well as for the L-NC group $[F(1,14)=8.23, p<.025]$. Improvement of performance obtained for the D-NC group did not reach an acceptable level of significance $[F(1,14)=3.67$, $p<.10$ ].

A two-way ANOVA (training condition $\times$ testing conditions) performed on the savings in avoidance indicated no effect of the training conditions (dark vs. light: $F<1$ ) and a significant main effect of the testing conditions [cued vs. noncued: $F(1,56)=11.34, p<.005$ ]; cued animals responded more than did noncued ones.

Paired comparisons of the savings in avoidance performed between cued and noncued animals indicated that pretest exposure to the light induced a significant improvement of the retention performance in the light condition $[F(1,28)=7.74, p<.025]$, as well as in the dark condition $[F(1,28)=4.37, p<.005]$.

\section{Discussion}

To summarize, the results of Experiment 1 demonstrated that a brightness discrimination is similarly acquired (despite some minor differences) and retrieved after a 1-day retention interval whether the darkened or the lighted arm is used as the safe arm. This supports Ray and Barrett's (1975) observations.

Furthermore, the results confirm that after a 1-day TTI, a pretest exposure to the light induces a large improvement of the subsequent retention performance in the light condition (Gisquet-Verrier \& Alexinsky, 1990a; GisquetVerrier et al., 1989). It further demonstrates that a pretest exposure to the light induces a similar improvement when delivered in the dark condition. The fact that an exposure to the light stimulus acts as an effective retrieval cue whether the animals had to reach the lighted arm or the darkened arm of the maze to avoid the electrical footshock suggests that the light affects the retention performance, not through its relation with safety or danger, but through its $C S$ value.

\section{EXPERIMENT 2}

Experiment 2 was aimed at testing the possibility that the lack of an additive effect among retrieval cues may result from a lack of coherence of the information conveyed by the cues delivered during the cuing treatment. For that purpose, the relative effectiveness of a pretest exposure to the grid, to the light, and to the grid and the light combined were investigated either in our usual light condition, in which animals had to reach the lighted arm, or in the dark condition, in which animals had to reach the darkened arm. According to our hypothesis, the reduced effectiveness of the compound cue will only be obtained when animals are trained in the light condition, where light is related to safety and grid features are related to electric shock, and not in the dark condition, because, in the latter, grid and light are both related to electric shock. On the other hand, if adding a feature to another disrupts the processing of the otherwise effective initial one, the compound cue will be ineffective in both training conditions.

We have already demonstrated, in the light condition, that after a 21 -day retention interval, exposure to the light is no longer effective as a reminder cue (Gisquet-Verrier et al., 1989). Exposure to the grid significantly improves the subsequent retention performance, whereas the combined exposure to the light and the grid has no effect. The aim of Experiment 2 was to replicate this finding and to test the respective effectiveness of the single cues relative to the compound cue in the dark condition.

\section{Method}

One hundred twenty-five naive male Sprague-Dawley rats maintained under the same conditions as those employed in Experiment 1 were used. Unless otherwise noted, the apparatus and procedure were the same as those used in Experiment 1.

The rats were randomly assigned to one of the two training conditions. Following pretraining and training, 5 rats were discarded because of their long response latencies at the end of training. The 120 remaining animals, 60 in each training condition (light and dark), were assigned to one of four groups $(n=15)$ on the basis of their training performance according to a 2 (training condition) $\times 4$ (pretest-cuing condition) factorial design. The two noncued groups of animals (L-NC and D-NC) were placed directly in the maze at the end of their 21-day TTI; the remaining animals were submitted to 2.5 -min pretest cuing, ending $5 \mathrm{~min}$ before the retention test, under the same conditions as those of Experiment 1.

Animals in the two BL groups (Groups L-BL and D-BL) were exposed to a series of five 2 -sec light flashes. In the two BG groups (Groups L-BG and D-BG), the rats were placed in the cuing box equipped with a grid floor identical to the floor of the $Y$-maze. Animals in the two BGL groups (Groups L-BGL and D-BGL) were placed in the cuing box equipped with the grid floor (the same as that for BG groups) and were further exposed to the five light flashes (as were BL groups). 


\section{Results}

Pretraining. An ANOVA performed on the number of visited arms during the 5-min period of pretraining indicated a significant effect of the training condition, with the animals placed in the dark condition visiting significantly more arms than those placed in the light condition $[M=21.07$ vs. $M=17.45 ; F(1,118)=5.64, p<.025]$.

Initial training. An ANOVA (training condition $\times$ trial block) revealed a significant main effect of trial block for each performance measure (response latencies, number of errors, and number of avoidance responses; $p<.001$, in every case). There were no differences between the two training conditions and no interaction between training conditions and trial blocks, regardless of the considered variable $(F \mathrm{~s}<1)$. At the end of training, no differences could be detected among the eight experimental groups $(F<1)$.
Cuing. A $2 \times 3$ ANOVA performed on the two activity measures indicated no differences among the two training conditions and the three cued groups. In the dark condition, there were no differences among the three cued groups of animals $[F(2,42)<1]$. In the light condition, a significant difference appeared among the three cued groups $[F(2,42)=3.42, p<.05]$, with the L-BL Group being more active than the L-BG and L-BGL groups $[F(1,28)=4.85, p<.025$, and $F(1,28)=4.15, p<$ .05 , respectively].

Retraining. Mean number of avoidance responses during training and retraining, as well as mean savings in avoidance, obtained for animals trained under $L$ and $D$ conditions are illustrated in Figure 3.

Effect of the training condition (D/L). An ANOVA performed on the five blocks of five retraining trials between the two noncued groups (L-NC and D-NC) re-
"L" CONDITION
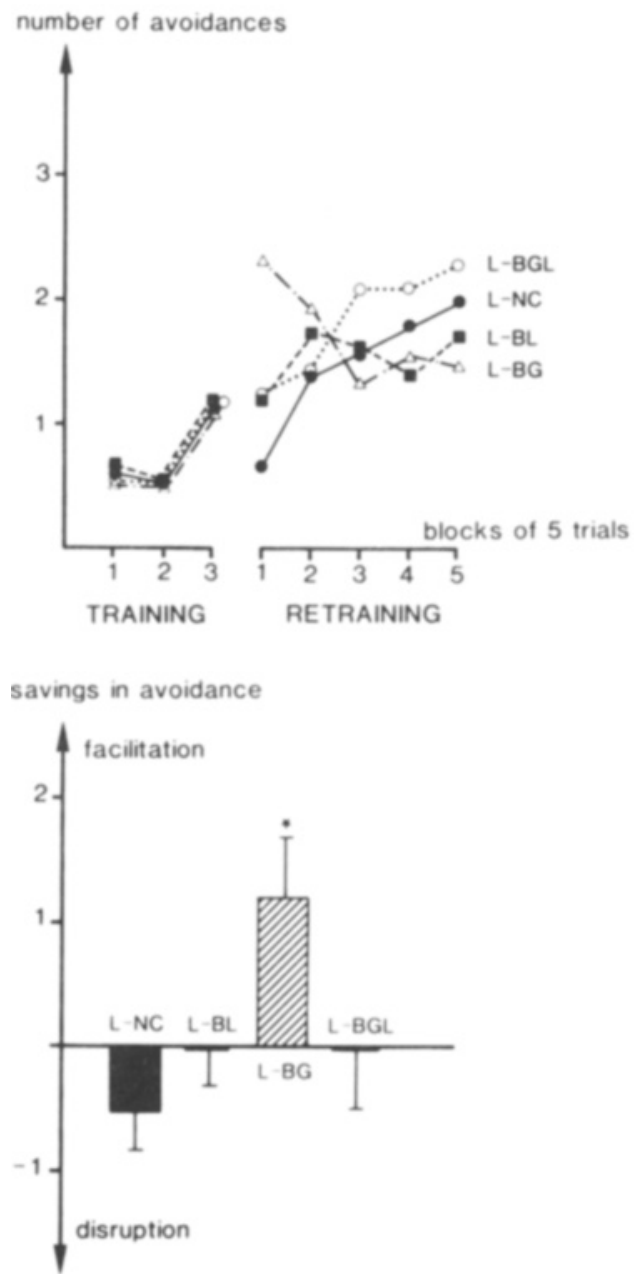

21 days TTI

"D" CONDITION
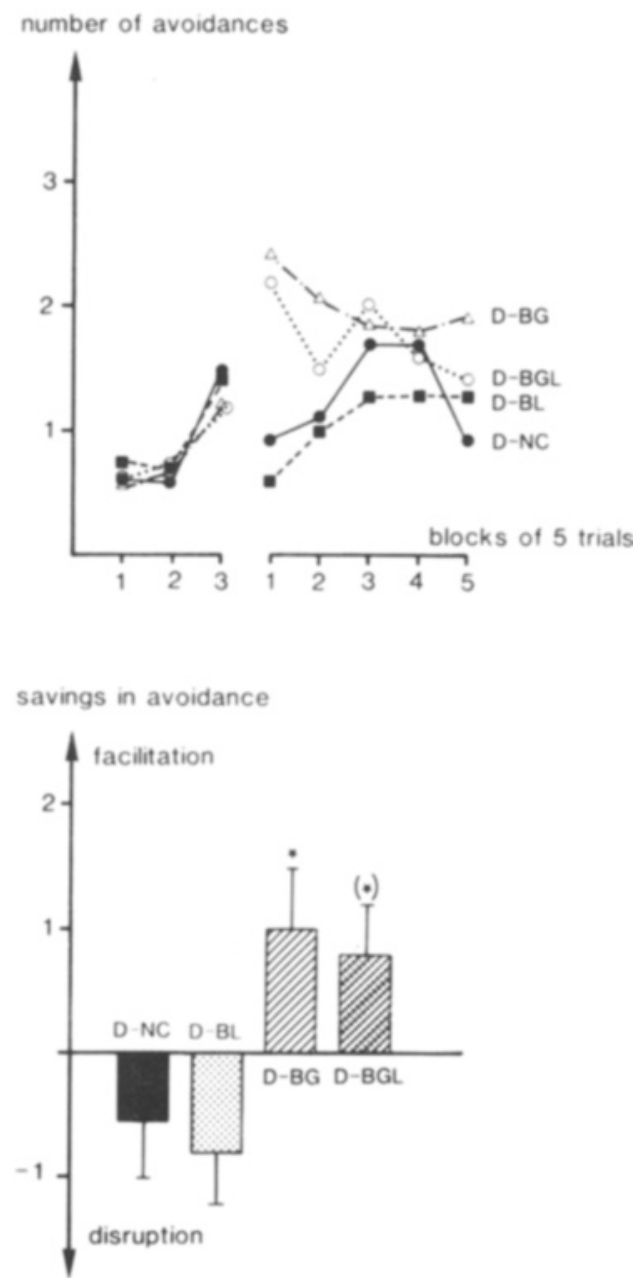

Figure 3. Upper: Mean number of avoidance responses (avoidance score) obtained during training and retraining in the light (L) and dark (D) training conditions for noncued animals (L-NC and D-NC), animals cued with the light (L-BL and D-BL), animals cued with the grid (L-BG and D-BG), and animals cued with the compound light plus grid (L-BGL and D-BGL). Lower: Mean ( \pm SEM) score of savings in avoidance obtained for each experimental group. Asterisks at the top of the columns correspond to within-group comparisons $[(*) .05<p<.10$, $* .025<p<.05$ ]. 
vealed no significant difference and no interaction between training condition and trial blocks, regardless of the considered variable: response latencies, number of errors, or number of avoidance responses.

Effect of pretest cuing: Retention performance. Within-group comparisons performed on the number of avoidance responses (avoidance score) indicated a significant improvement of performance between the end of training and the beginning of retraining for the $\mathrm{BG}$ Groups [Group L-BG, $F(1,14)=5.18, p<.05$; Group D-BG, $F(1,14)=4.67, p<.05]$. The disruption of performance obtained for the two noncued groups was not significant $[F(1,14)=2.58$ and $F(1,14)=1.37$, for Groups L-NC and D-NC, respectively]. Animals submitted to BL treatment did not show any significant alteration of the retention performance [Group L-BL, $F(1,14)=0$; Group D-BL, $F(1,14)=3.91, p<.10$ ]. Finally, Group D-BGL exhibited an improvement of performance that fell just short of significance $[F(1,14)=4.42, p<.07)$, and Group L-BGL did not show any modification of performance between training and testing $[F(1,14)=0]$.

In the light condition, paired comparisons on the savings in avoidance indicated that the noncued group did not significantly differ from Group L-BL or Group L-BGL $[F(1,28)=1.15$ and $F(1,28)=0$, respectively]. However, noncued animals exhibited significantly less savings in avoidance than did animals in Group L-BG $[F(1,28)=7.66, p<.005]$. The difference between Group L-BG and Group L-BGL was not significant $[F(1,28)=3.78, .05<p<.10]$.

In the dark condition, paired comparisons on the savings in avoidance indicated that the noncued group of animals (D-NC) did not significantly differ from those of Group D-BL $(F<1)$, but the noncued group did exhibit significantly less savings in avoidance than did Groups D-BG and D-BGL $[F(1,28)=5.5, p<.030$, and $F(1,28)=5.04, p<.045$, respectively]. Animals in Groups D-BG and D-BGL also significantly differed from animals in Group D-BL $[F(1,28)=8.46$, $p<.001$, and $F(1,28)=8.30, p<.010$, respectively].

Effect of pretest cuing: Reacquisition performance. In the light condition, the overall ANOVA (testing condition $\times$ trial block) performed on the number of avoidance responses (avoidance score) during retraining, among the four groups, indicated no significant effect of the testing conditions $(F<1)$, no significant effect of trial blocks $[F(4,224)=1.19]$, and no significant interaction between the two factors $[F(12,224)=1.73]$. However, paired comparisons indicated that animals in Group L-BG performed differently from those of all the remaining groups, as indicated by significant interactions between testing conditions and blocks of retraining trials $[\mathrm{L}-\mathrm{BG} / \mathrm{L}-\mathrm{BGL}$, $F(4,112)=4.18, p<.005 ; \mathrm{L}-\mathrm{BG} / \mathrm{L}-\mathrm{BL}, F(4,112)=$ $2.47, p<.05 ; \mathrm{L}-\mathrm{BG} / \mathrm{L}-\mathrm{NC}, F(4,112)=3.23, p<$ $.025]$. No significant interaction could be detected among the remaining groups, Groups L-BGL, L-BL, and L-NC $(F \mathrm{~s}<1$; see also Figure 3).
In the dark condition, the overall ANOVA (testing condition $\times$ trial block) performed on the number of avoidance responses during retraining demonstrated no significant effect of the testing conditions $[F(3,56)=$ 1.19], no significant effect of trial blocks $[F(4,224)=$ .93], and no significant interaction between the two factors $[F(12,224)=1.60]$. However, paired comparisons showed that there were significant interactions between testing conditions and blocks of retraining trials for Group D-BG and Group D-BL $[F(4,112)=2.42, p<.05]$, Group D-BG and Group D-NC $[F(4,112)=2.44$, $p<.05]$, and Group D-BGL and Group D-BL $[F(4,112)=2.46, p<.05]$. There was no interaction between trials for Group D-BGL versus Group D-NC $[F(4,112)=1.44$, n.s. $]$.

These data indicate that during reacquisition in the light condition, Group L-BG performed differently from the other groups, whereas in the dark condition, Group D-BG and Group D-BGL displayed a performance trend among retraining trials that differed from Group D-BL. Group D-BG also differed from Group D-NC (see also Figure 3).

Comparisons performed for each testing condition, between light and dark training conditions (training condition $\times$ trial block), on the number of avoidance responses during retraining indicated no effect of the training condition and no interaction between training condition and repetition of trials between Groups $\mathrm{L}-\mathrm{BL}$ and $\mathrm{D}-\mathrm{BL}$, Groups L-BG and D-BG, and Groups L-NC and D-NC ( $F \mathrm{~s}<1$, in every case). However, a significant interaction was obtained between Groups L-BGL and D-BGL $[F(4,112)=2.46, p<.05]$, indicating that the effect of treatment BGL clearly depends on the training condition (L or D).

\section{Discussion}

The results of Experiment 2 confirm that a brightness discrimination is similarly acquired when the animals are trained to avoid a footshock by reaching the lighted arm of a darkened maze or by reaching the darkened arm of a lighted maze. The results further demonstrate that in the absence of cuing these two versions of the same task are similarly retained after a 21-day TTI.

In the light condition, an exposure to the grid alone improved the subsequent retention performance, whereas joint exposure to the grid and the light did not affect the retention performance. In the dark condition, combined exposure to the light and the grid improved the subsequent retention performance, as mere exposure to the grid did. In both training conditions, and despite the fact that no significant interaction between testing conditions and blocks of retraining trials could be detected in overall comparisons, it appears that the animals avoiding most effectively at the beginning of testing (Groups L-BG, D-BG, and D-BGL) did not maintain their avoidance scores throughout the retraining session. We observed a similar tendency in other experiments (Gisquet-Verrier \& Alex- 
insky, 1990a), without being able to provide a convincing explanation for this phenomenon. Nevertheless, it appeared that during the retraining session, animals in Group L-BGL performed differently from those in Group L-BG, whereas animals in Group D-BGL performed similarly to those in Group D-BG. In all, these results show that light, when delivered in combination with grid, differentially affects the retrieval processes, depending on its significance in the training situation.

\section{GENERAL DISCUSSION}

Taken together, these experiments demonstrate that an avoidance brightness discrimination is similarly acquired whether the animals are trained to reach the lighted arm or the darkened arm as the safe arm of a Y-maze. These experiments further indicate no influence of the training condition either on motor activity during the cuing episode or on the retention performance after a 1-day or a 21-day retention interval.

The cuing effects obtained in the light training condition fully confirmed our previous results (Gisquet-Verrier \& Alexinsky, 1990a; Gisquet-Verrier et al., 1989). Most of the effects of prior cuing obtained in the dark condition are similar to those obtained in the light condition: A pretest exposure to the CS dramatically increased the retention performance when delivered relatively shortly after training (i.e., 1 day; Experiment 1), but it no longer had any effect when given after a 21-day TTI (Experiment 2). The fact that similar results are obtained when light indicates either a safe place or a place where an electric shock is delivered strongly suggests that light does not act through its relation with safety or danger but rather through its CS value, since this feature is kept constant in the two training conditions. A pretest exposure to the grid delivered long after training (i.e., 21 days; Experiment 2) not only compensated for the weak but reliable retention deficit due to long-term forgetting, but it significantly improved the retention performance. In the two versions of the task, the value of the grid is maintained since the electric shock is always delivered through the grid. These results suggest that when the significance of a particular feature is maintained, the effectiveness of this feature as a retrieval cue is preserved. These results clearly indicate that light and dark training conditions are equivalent not only in terms of learning processes but also in terms of susceptibility to retrieval cues.

The only difference obtained between the dark and light training conditions concerned the effects of the BGL compound cue, involving concurrent exposures to the grid and the light. Whereas this treatment was ineffective in the light condition, confirming our previous results (GisquetVerrier et al., 1989), it substantially improved the retention performance when delivered in the dark condition. The fact that the same compound treatment has different effects on the retention performance, depending on the training condition, precludes an interpretation of our results in terms of disruption of attentional processes (see
Deweer, 1986), since a similar disruptive effect would be expected in the two training conditions.

The present results showing that the effectiveness of a compound cue depends on the value of the light relative to that of the grid suggest that the coherence of information contained in the compound may be important: In the dark condition, the animals received an electric footshock in all lighted places of the maze. Exposing the rats to the grid induced an improvement of the retention performance, which was not affected by additional cuing exposures to the light, here related to presentation of the UCS, as was the grid. During the retraining session, animals exposed to the compound treatment behaved like those simply exposed to the grid.

In the light condition, the animals received an electric footshock in the whole maze, except in the lighted arm. Exposing the rats to the grid improved the subsequent retention performance. However, additional exposure to the light, which in this situation indicated safety, abolished the cuing effectiveness of the grid. Furthermore, during retraining, rats exposed to the compound cue exhibited performance that significantly differed from that of rats exposed to the grid alone.

Hence, when exposure to a cue is able to enhance the subsequent retention performance, adding supplementary information during cuing may either have no effect, when the latter information is compatible with the initial information, or have a detrimental effect, when the added information is not compatible with the former.

As an illustration of the "coherence between cues" hypothesis, we observed in previous experiments (GisquetVerrier et al., 1989) that, after a 21-day TTI, alternate presentation of the light and the footshock did not act as a retrieval cue, whereas an ordered sequence of these same training features (light on, no footshock, light off followed by a footshock) was a very potent retrieval cue. When delivered alternately, the two training features were unable to promote the retrieval processes adequately, but when delivered in a way preserving the value of a particular feature relative to the other, the same training features were very effective. In the same experiment, we repeatedly observed that an exposure to the grid alone induced similar effects on the retention performance as an exposure to the grid plus the electric footshock (see also Spear \& Parsons, 1976). There is convincing evidence in the literature that exposure to environmental stimuli previously paired with an electric footshock produces peripheral and central effects similar to the stressful event itself (Bryan \& Lehman, 1988; Cassens, Roffman, Kuruc, Orsulak, \& Schildkraut, 1980). These previous results indicated no simple additive effects between cues. They support the coherence hypothesis in showing that exposure to an additional training feature during prior cuing does not alter the initial effectiveness of a particular training feature as a retrieval cue, when the additional cue is coherent with the initial one.

The present results suggest that a particular feature may be analyzed differently, depending on whether it is de- 
livered alone or in combination with another feature. When presented alone after a 1-day TTI, light was an effective retrieval cue in both training conditions, suggesting that light acts through its CS value. The fact that, after a 21-day TTI, addition of the light in a cuing treatment with the grid differentially alters the cuing effectiveness of the grid alone (depending on the training condition) suggests that when delivered in combination with grid, light acts through its relation with safety or danger. As an explanation, one might suggest that the same training feature light is taken into account differently, depending on whether it is shortly after training or long after training. This would explain why exposure to the same training feature may differentially affect the retrieval processes, depending on the length of the TTI (Gisquet-Verrier et al., 1989). However, since similar incompatibility between light and grid was observed as soon as after a 1-hour TTI, this hypothesis seems unlikely. An alternative possibility is that the value of an additional cue is analyzed in relation to the initial cue. Since, in the compound treatment, animals are first exposed to the grid before being exposed to the light, perhaps the value of the light is analyzed relative to that of the grid. Hence, whereas light alone acts through its CS value, light in combination with grid would be analyzed as indicating safety in the light condition and danger in the dark condition. This would account for the differential effectiveness of the same BGL compound treatment in the light and dark conditions in the present experiments.

The fact that coherence between training features may determine the effectiveness of a compound cue can account for the inconsistency of the data obtained in the few studies that have investigated possible additive effects of retrieval cues (Devietti \& Hopfer, 1974; Deweer, 1986; Moye \& Thomas, 1982; Spear \& Parsons, 1976).

The facilitative effect of prior cuing on retention performance is generally considered as being due to the reactivation of the initial memory trace triggered by the reactivation of a certain number/kind of memory attributes, internal representations of the different training features to which the subject was exposed (R. R. Miller, Kasprow, \& Schachtman, 1986; Spear, 1978). Hence, direct reactivation is generally inferred as resulting from prior cuing, because of the animal's overt behavior. The fact that exposure to the compound cue did not induce any performance improvement during the subsequent retention test in animals trained in the light condition could then be due to a lack of reactivation of the corresponding memory attributes. This would imply that incoherence between training features has to be detected at once, without any analysis of the features, which seems unlikely. The other possibility is that exposure to the compound cue induces the reactivation of the corresponding attributes, but that despite a partial memory reactivation larger than the one induced with simple cues, reactivation of the whole memory trace does not occur. Such a failure could be due to the coactivation of different memory attributes that must not be activated simultaneously because they are related, for instance, to opposite behavioral responses.
The present results support the contention that multiple pretest cues are not merely additive in their effect (Spear, J. S. Miller, \& Jagliego, 1990). The results of these experiments, as well as previous ones (GisquetVerrier \& Alexinsky, 1990a; Gisquet-Verrier et al., 1989), lead us to emphasize that when an organism is faced with compound cues, reactivation processes may function adequately but retrieval of a given memory may fail: Such ineffective retrieval may occur whenever the subject notices different events during cuing that correspond to and, hence, evoke attributes that lead to incompatible behavioral responses.

\section{REFERENCES}

Bryan, R. M., \& Lehman, R. A. W. (1988). Cerebral glucose utilization after aversive conditioning and during conditioned fear in the rat. Brain Research, 444, 17-24.

Cassens, G., Roffman, M., Kuruc, A., Orsulak, P. J., \& SchildKRAUT, J. J. (1980). Alteration in brain norepinephrine metabolism induced by environmental stimuli previously paired with inescapable shock. Science, 209, 1138-1139.

Devietti, T. L., \& Hopfer, T. M. (1974). Complete amnesia induced by ECS and complete recovery of memory following reinstatement treatment. Physiology \& Behavior, 12, 599-603.

DEWEER, B. (1986). Pretest cuing after forgetting of a food-motivated maze task in rats: Synergistic action of context and reinforcement. Animal Learning \& Behavior, 14, 249-256.

Deweer, B., Sara, S. J., \& Hars, B. (1980). Contextual cues and memory retrieval in rats: Alleviation of forgetting. Animal Leaming \& Behavior, 8, 265-272.

Gisquet-Verrier, P., \& Alexinsky, T. (1986). Does contextual change determine long-term forgetting? Animal Learning \& Behavior, 14, 349-358.

Gisquet-Verrier, P., \& Alexinsky, T. (1988). Time-dependent fluctuations of retention performance in an aversively motivated task. Animal Learning \& Behavior, 16, 58-66.

Gisquet-VerRIER, P., \& AleXINSKy, T. (1990a). Facilitative effect of a pretest exposure to the CS: Analysis and implications for the memory trace. Animal Learning \& Behavior, 18, 323-331.

Gisquet-VerRIER, P., \& AleXinSKY, T. (1990b). Long-term spontaneous improvement of performance is related to the strength of the initial training: Theoretical implications. Behavioral \& Neural Biology, 53, 298-304

Gisquet-Verrier, P., Dekeyne, A., a Alexinsky, T. (1989). Differential effects of several retrieval cues over time: Evidence for timedependent reorganization of memory. Animal Learning \& Behavior, 17, 394-408.

Gisquet-Verrier, P., \& Smith, C. (1989). Avoidance performance in rat enhanced by post-learning paradoxical sleep deprivation. Behavioral \& Neural Biology, 52, 152-169.

Gordon, W. C. (1981). Mechanisms of cue-induced enhancement. In N. E. Spear \& R. R. Miller (Eds.), Information processing in animals: Memory mechanism (pp. 319-339). Hillsdale, NJ: Erlbaum.

Gordon, W. C., F Feldman, D. T. (1978). Reactivation-induced interference in a short-term retention paradigm. Learning \& Motivation, $9,164-178$.

KLEIN, S. B., \& SPEAR, N. E. (1970). Reactivation of avoidance-learning memory in the rat after intermediate retention interval. Journal of Comparative \& Physiological Psychology, 72, $498-504$.

KoppenaAl, R., Jagoda, E., \& CRUCE, J. A. S. (1967). Recovery from ECS-produced amnesia following a reminder. Psychonomic Science, 9, 293-294.

LEWIS, D. J. (1979). Psychobiology of active and inactive memory. Psychological Bulletin, 86, 1054-1083.

Lewis, D. J., MisAnin, J. R., Miller, R. R. (1968). Recovery of memory following amnesia. Nature, 220, 704-705.

Miller, J. S., Jagielo, J. A., \& SPEar, N. E. (1991). Differential effectiveness of various prior-cuing treatments in the reactivation and 
the maintenance of memory. Journal of Experimental Psychology: Animal Behavior Processes, 17, 249-258.

Miller, R. R., Kasprow, W. J., \& Schachtman, T. R. (1986). Retrieval variability: Sources and consequences. American Joumal of Psychology, 99, 145-218.

Miller, R. R., \& SPRINGER, A. D. (1972). Induced recovery of memory in rats following electroconvulsive shock. Physiology \& Behavior, 8, 645-651.

Moye, T. B., \& Thomas, D. R. (1982). Effects of memory reactivation treatments on postdiscrimination generalization performance in pigeons. Animal Learning \& Behavior, 10, 159-166.

RAY, O. S., \& BARRETT, R. J. (1975). Behavioral and biochemical analysis of genetic differences in rats. Behavioral Biology, 15, 391-417.

Richardson, R., Riccio, D. C., \& Axiotis, R. (1986). Alleviation of infantile amnesia in rats by internal and external contextual cues. $D e$ velopmental Psychobiology, 19, 453-462.

Rovee-Collier, C., \& HAYNe, H. (1987). Reactivation of infant memory: Implications for cognitive development. Advances in Child Development Behavior, 20, 185-238.
SARA, S. J. (1973a). Progressive development of avoidance response after training, ECS and repeated testing. Bulletin of the Psychonomic Society, 2, 134-136.

SARA, S. J. (1973b). Recovery from hypoxia and ECS induced amnesia after a single exposure to the training environment. Physiology \& Behavior, 9, 85-89.

SPEAR, N. E. (1978). The processing in memories: Forgetting and retention. Hillsdale, NJ: Erlbaum.

Spear, N. E., Miller, J. S., \& Jagielo, J. A. (1990). Animal memory and learning. Annual Review of Psychology, 41, 169-211.

SPEAR, N. E., \& PARSONS, P. (1976). Analysis of a reactivation treatment: Ontogeny and alleviated forgetting. In R. Davies \& W. Roberts (Eds.), Coding processes in animal memory (pp. 135-165). Hillsdale, NJ: Erlbaum.

Underwood, B. J. (1969). Attributes of memory. Psychological Review, 67, 73-95.

(Manuscript received November 11, 1991; revision accepted for publication June 23,1992 .) 